\title{
Active search about latex in injectable drugs for patient's safety in medical therapy
}

\author{
Roberta Guerra PEIXE' ${ }^{1}$ (D) Maria Clara SODRÉ ${ }^{1}$ (D), Beatriz Bortolami OLIVEIRA ${ }^{1}$ (D), Luana Perazio GONÇALVES ${ }^{1}$ (D) lan Giacometti HALM $^{1}$ (D), \\ José Carlos CRUZ² (D), Ana Paula ANTUNES² (D), Rodrigo Teixeira AMANCIO² (D)
}

${ }^{1}$ Universidade Federal do Rio de Janeiro, Niterói - RJ, ${ }^{2}$ Hospital Federal dos Servidores do Estado, Rio de Janeiro - RJ

Corresponding author: Peixe, RG, robertapeixe@gmail.com

Submitted: 09-10-2021 Resubmitted: 01-12-2021 Accepted: 01-12-2021

Peer review: blind reviewers

\begin{abstract}
Objectives: To classify injectable drugs used in a general hospital inventory according to latex presence or absence in vials with rubber stoppers, antibiotics, and electrolytes bags. Methods: The information about latex content has been collected from medicine industries, after identification of authorized manufacturers in Brazil, through the National Health Surveillance Agency (ANVISA) medicine search database. Medicines have been grouped per pharmaceutical manufacturers, and data were collected through telephone or e-mail. Only written information from pharmaceutical companies were considered. Results: Among 161 medicines used in the hospital packaged in vials with rubber stoppers, antibiotics, and electrolytes bags, 123 (76.4\%) medicine-related responses were received, covering 540 (82.2\%) different presentations. For this, from 87 contacts, 70 (80.5\%) companies responded and 65 (74.7\%) provided information related to the presence or absence of the allergen. Among the medicine list, 8 (6.5\%) were identified as containing latex (in 12 different presentations $-2.2 \%$ ) and 101 medications (82.1\%) in 507 presentations (93.9\%) do not contain latex. The manufacturers responded that the medicine was latex-free, but could not confirm for raw materials ( 6 medicines - 4.9\%- in 6 different presentations $-1.1 \%$ ) and that they were unable to ensure there was no contact with latex during manipulation (4 medicines $-3.3 \%$ - in 8 different presentations - $1.5 \%$ ). For 4 medicines (3.3\%) in 7 different presentations (1.3\%), the companies were inconclusive, as they could not confirm whether they were latex-free or not. Conclusion: Although latex is a known allergen, information about the presence of latex components in pharmaceutical packaging and package inserts needs improvement, since the active search identified 161 medicines packaged in vials with rubber stoppers, antibiotics, and electrolytes bags that failed to depict this information. The results of this survey endorse the importance of displaying this information on medicines label or packaging in order to ensure healthcare professionals easy access and prompt consultation.
\end{abstract}

Keywords: injectable medicines; latex rubber; patient safety; latex hypersensitivity; latex allergy; drug packaging.

\section{Busca ativa sobre a presença de látex em medicamentos injetáveis para a segurança do paciente na terapia medicamentosa}

\section{Resumo}

Objetivos: Classificar os medicamentos injetáveis padronizados em um hospital geral do Rio de Janeiro, de acordo com a presença ou ausência de látex acondicionados em frascos de medicamentos, bolsas de antibióticos e eletrólitos. Métodos: As informações sobre a presença ou ausência de látex nas embalagens dos medicamentos foram coletadas junto às indústrias farmacêuticas, após verificação dos fabricantes com registro ativo no Brasil, através da plataforma de consulta a medicamentos da ANVISA. Os medicamentos foram agrupados por fabricantes e foi realizado contato pelos canais disponibilizados pelo fabricante para obter as informações necessárias. Foram consideradas válidas apenas informações recebidas por escrito. Resultados: Dentre os 161 medicamentos padronizados acondicionados em frascos, bolsas de antibióticos e eletrólitos, foram recebidas respostas relacionadas a 123 medicamentos (76,4\%), em 540 diferentes apresentações (82,2\%). Das 87 empresas contatadas, 70 (80,5\%) responderam aos pesquisadores e 65 (74,7\%) disponibilizaram as informações relacionadas a presença ou ausência do alérgeno. Dentre a lista de medicamentos, 8 (6,5\%) contém látex (em 12 diferentes apresentações -2,2\%) e 101 (82,1\%) não contém látex (em 507 diferentes apresentações-93,9\%). Foram obtidas respostas onde o fabricante afirmou que o medicamento não continha látex, porém não poderia confirmar que suas matérias-primas estavam livres do alérgeno ( 6 medicamentos $-4,9 \%$ - em 6 diferentes apresentações - 1,1\%) e que não garantiam que durante a manipulação do medicamento não poderia haver o contato com látex (4 medicamentos - 3,3\%- em 8 diferentes apresentações -1,5\%). Em 4 medicamentos (3,3\%), em 7 apresentações diferentes (1,3\%), a resposta das empresas foi inconclusiva, onde não poderiam confirmar nem a ausência nem a presença de látex. Conclusão: Embora o látex seja um alérgeno conhecido, as informações sobre a existência ou não do látex nas informações dos fabricantes de produtos farmacêuticos carece de melhora na qualidade da informação, uma vez que nossa busca ativa identificou 161 medicamentos acondicionados em frascos, bolsas de antibióticos e eletrólitos que não apresentavam estas informações de maneira sistematizada. Os resultados desse levantamento reforçam a importância dessa informação estar contida na bula ou embalagem dos medicamentos, de maneira a garantir acesso facilitado e para rápida consulta por parte das equipes de saúde.

Palavras-chave: medicamentos injetáveis; borracha de látex; segurança do paciente; sensibilidade ao látex; alergia ao látex; embalagem de medicamentos. 


\section{Introduction}

Latex is an important raw material utilized worldwide as a component of many medical devices as gloves, condoms, packages, vials, and stoppers. Extracted from the tree Hervea brasiliensis, latex is a milky fluid consisting of nucleotides, proteins, lipids, and polypeptides. Among the polypeptides identified so far, 56 are allergenic and may cause sensitivity and/or allergy in patients. The sensitivity process may develop after repeated exposure periods from six months to 15 years. ${ }^{1-4}$ Latex reactions can occur as dermatitis and urticaria, possibly leading to life-threatening allergic reactions. . $^{5,6}$

Data about allergy and allergen sensitivity are scarce, few studies address reactions in health professionals and patients with special cases $^{7,8}$. Furthermore, patients submitted to several surgical procedures and workers of the latex industry are examples of the sensitive risk group ${ }^{6,9}$. Latex sensitiveness affects from $1 \%$ to $6 \%$ of the population and from $8 \%$ to $12 \%$ of health professionals who expose themselves usually to allergen-containing materials., ${ }^{1,3}$ Among anesthesiologists, the incidence of allergy is $12.5 \%$ to $15.8 \% .{ }^{10}$ In Brazil, Montalvão et al (2008) conducted a study in a general hospital, where the prevalence of professionals with latex allergy was 3.7\%. ${ }^{11}$ Gomes et al (2012) showed that $22.4 \%$ of dental, medical and nursing students from a Federal University were sensitive to latex. ${ }^{3}$ At a teaching hospital, the latex allergy rate was $1.02 \%$ among health professionals. ${ }^{12}$

The most susceptible patients who submit to surgical procedures and catheterization, if exposed to latex allergen, can develop allergic reactions. The prevalence of latex allergy in these patients is $7.2 \%$ and sensitiveness is $30.4 \%{ }^{1,3}$ Yeh et al (2012), demonstrated a higher number of readmissions of Brazilians patients who suffer from myelomeningocele and latex allergy compared to others with the same pathology, but not allergic. ${ }^{13}$ Sá et al (2013), detected the prevalence of $25 \%$ of sensitization and $20 \%$ of allergy to latex in children and adolescents with myelomeningocele. ${ }^{14}$ Garro et al (2017) observed sensitization in 33.2\% and allergy in $12.2 \%$ of patients with neural tube defect. ${ }^{15}$

Since its creation in 2013, the National Patient Safety Program (PNSP) helps health professionals to prioritize the care to the patient with positive actions to improve its quality. ${ }^{16}$ From the six PNSP goals, the third which addresses the safety improvement on the medical prescription and medication administration, also encompasses the management of allergies related to adverse drug reactions. Medications, inputs, and other substances which may induce allergies should be included in the medical chart and prescription to help the health team. ${ }^{17}$

The contamination of medicines and vaccines with latex traces is a reality that can cause risk to allergic patients. ${ }^{1,3}$ The US Centers for Disease Control and Prevention within a world effort, endeavors to list the presence of allergens in vaccines. Surveys conducted in hospital pharmacies attempted to help and manage the active search for allergic patients. ${ }^{10,18}$ Interdisciplinary action is required to prevent accidents with the allergen through routines adjustments, standardization, and easily accessible information for patient safety and improved care. Since 2013 in Brazil, product manufacturers are required by law to warn consumers about the presence of natural latex in their packaging. ${ }^{19}$ ANVISA - Brazilian National Health Surveillance Agency, in College Board Resolution (RDC) number 26/2015, regulates the mandatory food labeling requirements mainly involved in allergies, including those which may contain latex. ${ }^{20}$ However, there is no regulation for compliance with the Law on medicines.
It is challenging to keep an updated database of latex containing medications and inputs because different manufacturers have different primary packages for the same medication or traces of latex in their industrial facilities. ${ }^{7}$ These are not easily accessible information as they are not included in the packages inserts or products packaging. ${ }^{21}$ It is essential to elaborate a drug database for medication information, promoting the safety culture and preventing latex-related reactions in hospitals.

Thus, we aimed to identify and classify injectable drugs used in a general hospital according to the presence or absence of latex in rubber stoppers, antibiotics, and electrolytes.

\section{Methods}

This is a retrospective, descriptive study of the identification process and classification of drugs packaged in vials with rubber stoppers, antibiotics, and electrolytes bags present in the hospital inventory regarding the presence or absence of latex carried out from August 2019 to September 2020. The study was developed by resident pharmacists and their Pharmacy preceptors at the Hospital Federal dos Servidores do Estado (HFSE), a general hospital from Rio de Janeiro, Brazil.

Standard drugs used in HFSE, packed in vials or plastic bags were the study base. We collected all the data provided by the pharmaceutical industry manufacturers. As there are various manufacturers because of the public bidding process, the active principles were searched at the ANVISA website where all the information about registered pharmaceutical manufacturers in Brazil is gathered. ${ }^{22}$

The drugs were grouped according to their respective pharmaceutical lab and the contact channel with these to obtain the required information, such as telephone and e-mail. Only written information were considered valid. We used Microsoft Excel $^{\circ}$ to tabulate all data collected according to the drug, presentation, manufacturer and presence or absence of latex in the package. Drugs were grouped alphabetically according to the Brazilian Common Denomination (DCB). The presence or absence of latex was classified as: 1) contains latex; 2) does not contain latex; 3 ) does not contain latex, but unable to ensure that during the manipulation no traces of the allergen are found; 4) does not contain latex, but unable to ensure whether raw materials contain the allergen; 5) does not contain latex, but unable to ensure whether allergen-free. During the analysis of our results, we excluded all drugs related to pharmaceutical manufacturers who did not reply to our requests and were unable to contact us.

The verification process for standard drugs at the HFSE, packaged in vials with rubber stoppers, antibiotics, and electrolytes bags, was carried out by five resident pharmacists and checked with the Pharmacy preceptors. The list of drugs was divided among the five residents, who searched ANVISA for their manufacturers. To ensure the reliability of the process, a pharmacist was responsible for sending all emails to pharmaceutical industries and distributors, in addition to compiling all the responses obtained. At the end of the compilation of the collected data, another pharmacist checked all the answers obtained through the emails received and crossed them with the results obtained, preventing errors or omissions from occurring at the end of the study.

The study was approved by the Institutional Review Board of HFSE (CAAE 41262620.7.0000.5252 on December 23 ${ }^{\text {th }} 2020$. 


\section{Results}

We identified 161 drugs packaged in vials with rubber stoppers, antibiotics, and electrolytes bags. After a survey at ANVISA website, 657 registered presentations of 87 different manufacturers were identified. Seventy (80.5\%) manufacturers responded when contacted, 65 (74.7\%) through e-mail containing latex-related responses. Information from 540 (82.2\%) different presentations of 123 drugs (76.4\%) used in the hospital have been received (Figures 1 and 2).

One hundred one drugs (82.1\%) in 507 presentations (93.9\%) do not contain latex. 8 drugs (6.5\%) were detected in 12 presentations $(2.2 \%)$ with latex. Four manufacturers providing 4 drugs $(3.3 \%)$ in 8 different presentations (1.5\%) reported their medications are latexfree. Nevertheless, they are unable to ensure that any latex traces can be found during the manipulation process. Three manufacturers distributing 6 drugs (4.9\%) with 6 different presentations (1.1\%) reported their medications are latex-free, they are not sure whether raw materials are allergen-free as well. Finally, three different manufacturers of 4 drugs (3.3\%) in 7 different presentations (1.3\%) affirmed their medications are latex-free, but also, are unable to ensure its absence (Figures 3 and 4).
Figure 1. The results obtained after an assessment of the information received from pharmaceutical manufacturers of standard drugs used in the hospital.

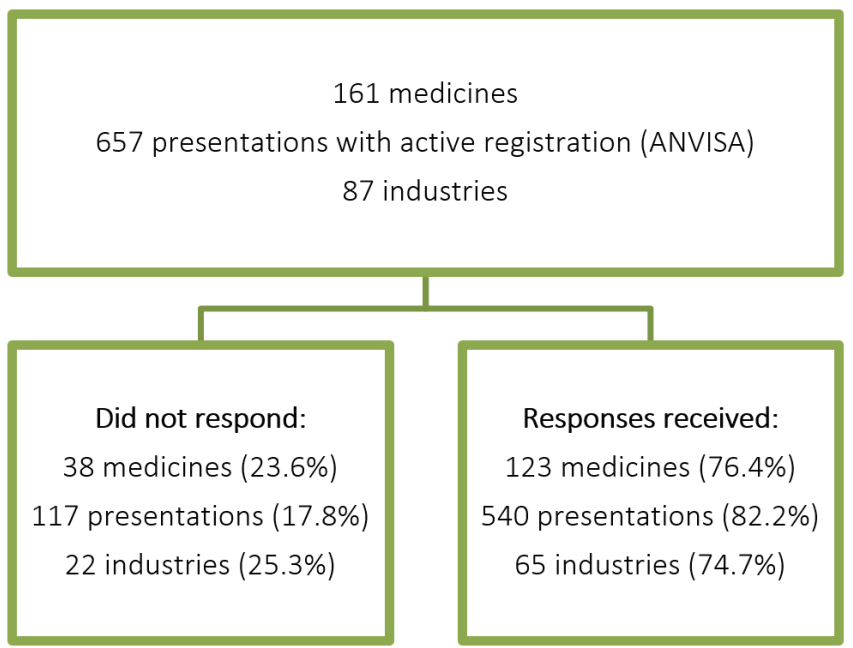

Figure 2: Information about the presence of latex in standard injectable drugs.

\begin{tabular}{|c|c|c|c|}
\hline Drugs & Presentation & Manufacturer & Does it contain latex? \\
\hline \multirow{5}{*}{ Acyclovir } & Zynvir 250 mg F/A & Novafarma & No \\
\hline & Acyclovir 250 mg F/A & Teuto & No \\
\hline & Ezopen 250 mg F/A & Teuto & No \\
\hline & Acyclovir $250 \mathrm{mg} F / \mathrm{A}$ & União Química & No \\
\hline & Uni-vir 250 mg F/A & União Química & No \\
\hline Alanylglutamine & Dipeptiven 20\% F/A 100 mL & Fresenius Kabi & No \\
\hline Alfaepoetin & Alfaepoetin 4.000 UI F/A 1 mL & Blau & No \\
\hline Alprostadil & Alprostadil $20 \mathrm{mcg} F / \mathrm{A}$ & Flukka & No \\
\hline \multirow{2}{*}{ Alteplase } & Actilyse $10 \mathrm{mg} F / \mathrm{A}$ & Boehringer Ingelheim & Yes \\
\hline & Actilyse $50 \mathrm{mg} F / \mathrm{A}$ & Boehringer Ingelheim & Yes \\
\hline \multirow{3}{*}{ Amoxicillin + clavulanate } & Amoxicillin + clavulanate $1 \mathrm{~g}+200 \mathrm{mg} \mathrm{F} / \mathrm{A}$ & Aurobindo & No \\
\hline & Amoxicillin + clavulanate $1 \mathrm{~g}+200 \mathrm{mg}$ F/A & Blau & No \\
\hline & Amoxicillin + clavulanate $1 \mathrm{~g}+200 \mathrm{mg}$ F/A & Teuto & No \\
\hline Amphotericin B & Anphotericin B 50 mg F/A & Cristália & No \\
\hline Amphotericin B lipid compound & Abelcet 5 mg/mL F/A 20 mL & Teva & No \\
\hline Amphotericin B liposomal & Ambisome $5 \mathrm{mg} / \mathrm{mL}$ F/A $10 \mathrm{~mL}$ & United Medical & No \\
\hline \multirow{5}{*}{ Ampicillin } & Ampicillin $1 \mathrm{~g}$ F/A & Aurobindo & No \\
\hline & Cilinon $1 \mathrm{~g} F / \mathrm{A}$ & Blau & No \\
\hline & Amplatil 1 g F/A & Novafarma & No \\
\hline & Ampicillin $1 \mathrm{~g} F / \mathrm{A}$ & Teuto & No \\
\hline & Teuplafec 1 g F/A & Teuto & No \\
\hline \multirow{3}{*}{ Ampicillin + sulbactam } & Ampicillin + sulbactam $2 \mathrm{~g}+1 \mathrm{~g} F / \mathrm{A}$ & Aurobindo & No \\
\hline & Ampicillin + sulbactam $2 \mathrm{~g}+1 \mathrm{~g}$ F/A & Eurofarma & No \\
\hline & Ampicillin + sulbactam $1 \mathrm{~g}+500 \mathrm{mg}$ F/A & Eurofarma & No \\
\hline \multirow{2}{*}{ Azacitidine } & Winduza $100 \mathrm{mg} F / \mathrm{A}$ & Dr Reddy's & No \\
\hline & Azacitidine $100 \mathrm{mg} F / \mathrm{A}$ & Dr Reddy's & No \\
\hline \multirow{3}{*}{ Azithromycin } & Azithromycin 500 mg F/A & Cristália & No \\
\hline & Azicin 500 mg F/A & Cristália & No \\
\hline & Astro IV 500 mg F/A & Eurofarma & No \\
\hline
\end{tabular}


Figure 2: Information about the presence of latex in standard injectable drugs.

\begin{tabular}{|c|c|c|c|}
\hline Drugs & Presentation & Manufacturer & Does it contain latex? \\
\hline \multirow[t]{2}{*}{ Benzathine benzylpenicillin } & Benzetacil 1.200.000 UI F/A & Eurofarma & $\begin{array}{l}\text { No, but unable to ensure raw materials } \\
\text { are latex-free }\end{array}$ \\
\hline & Benzathine benzylpenicillin 1.200.000 UI F/A & Teuto & No \\
\hline \multirow{2}{*}{ Benzylpenicillin procaine } & Penkaron 400.000 UI F/A & Blau & No \\
\hline & Wonilin 400.000 UI F/A & Novafarma & No \\
\hline Bevacizumab & Avastin 25 mg/mL F/A 4 mL & Roche & No, but unable to ensure is latex-free \\
\hline \multirow{7}{*}{ Bortezomib } & Bortezomib 3.5 mg F/A & Accord & No \\
\hline & Bortyz 3.5 mg F/A & Accord & No \\
\hline & Mielocade $3,5 \mathrm{mg} F / \mathrm{A}$ & Bérgamo & No \\
\hline & Bortezomib 3,5 mg F/A & Bérgamo & No \\
\hline & Bortezomib 3,5 mg F/A & Dr Reddy's & No \\
\hline & Bozored 3,5 mg F/A & Dr Reddy's & No \\
\hline & Velcade $3,5 \mathrm{mg} F / \mathrm{A}$ & Janssen-Cilag & No \\
\hline \multirow[t]{2}{*}{ Botulinum toxin $\mathrm{A}$} & Botox 100 UI F/A & Allergan & $\begin{array}{l}\text { No, but unable to ensure that during } \\
\text { manipulation no latex traces are found }\end{array}$ \\
\hline & Botulim 100 UI F/A & Blau & No \\
\hline Bovine heparin & Heptar $5.000 \mathrm{UI} / \mathrm{mL}$ F/A $5 \mathrm{~mL}$ & Eurofarma & No \\
\hline Bupivacaine & Neocaine $0.75 \%$ F/A $20 \mathrm{~mL}$ & Cristália & No \\
\hline \multirow{3}{*}{ Bupivacaine } & Neocaine $0.5 \%$ F/A 20 mL & Cristália & No \\
\hline & Bupivacaine $0,5 \% \mathrm{~F} / \mathrm{A} 20 \mathrm{~mL}$ & Hypofarma & No \\
\hline & Bupstesic 0.5\% F/A 20 mL & União Química & No \\
\hline Bupivacaine with vasoconstrictor & Neocaine $0.5 \%$ F/A $20 \mathrm{~mL}$ & Cristália & No \\
\hline \multirow{2}{*}{ Cabazitaxel } & Cabazitaxel 60 mg F/A & Dr Reddy's & No \\
\hline & Cabazred 60 mg F/A & Dr Reddy's & No \\
\hline \multirow{2}{*}{ Cabazitaxel } & Proazitax 60 mg F/A & Eurofarma & No \\
\hline & Jevtana $60 \mathrm{mg} F / \mathrm{A}$ & Sanofi & No \\
\hline \multirow{3}{*}{ Calcium folinate } & Calcium folinate $10 \mathrm{mg} F / \mathrm{A}$ & Eurofarma & No \\
\hline & Calcium folinate $50 \mathrm{mg} \mathrm{F} / \mathrm{A}$ & Eurofarma & No \\
\hline & Fauldleuco 50 mg F/A & Libbs & No \\
\hline Carbacol & Ophtcol 0,1 mg/mL F/A $2 \mathrm{~mL}$ & Ophthalmos & No \\
\hline \multirow{12}{*}{ Carboplatin } & Carboplatin 150 mg F/A & Accord & No \\
\hline & Carboplatin 450 mg & Accord & No \\
\hline & B-platin 150 mg F/A & Blau & No \\
\hline & B-platin 450 mg F/A & Blau & No \\
\hline & Evocarb 150 mg F/A & Farmarin & No \\
\hline & Evocarb 450 mg F/A & Farmarin & No \\
\hline & Displata 150 mg F/A & Fresenius Kabi & No \\
\hline & Displata 450 mg F/A & Fresenius Kabi & No \\
\hline & Fauldcarbo 150 mg F/A & Libbs & No \\
\hline & Fauldcarbo 450 mg F/A & Libbs & No \\
\hline & Tevacarbo 150 mg F/A & Teva & No \\
\hline & Tevacarbo 450 mg F/A & Teva & No \\
\hline Caspofungin & Cancidas 50 mg F/A & MSD & No \\
\hline \multirow{9}{*}{ Cefazolin } & Cefazolin $1 \mathrm{~g} \mathrm{~F} / \mathrm{A}$ & $A B L$ & No \\
\hline & Kefazol $1 \mathrm{~g}$ F/A & $A B L$ & No \\
\hline & Cefazolin $1 \mathrm{~g}$ F/A & Aurobindo & No \\
\hline & Cezolin $1 \mathrm{~g}$ F/A & Instituto Biochimico & No \\
\hline & Cefazolin $1 \mathrm{~g} \mathrm{~F} / \mathrm{A}$ & Instituto Biochimico & No \\
\hline & Fazolon $1 \mathrm{~g} \mathrm{~F} / \mathrm{A}$ & Blau & No \\
\hline & Fazolix $1 \mathrm{~g} \mathrm{F/A}$ & Novafarma & No \\
\hline & Cefazolin $1 \mathrm{~g} \mathrm{~F} / \mathrm{A}$ & Novafarma & No \\
\hline & Cefazolin 1 g F/A & União Química & No \\
\hline
\end{tabular}


Figure 2: Information about the presence of latex in standard injectable drugs.

\begin{tabular}{|c|c|c|c|}
\hline Drugs & Presentation & Manufacturer & Does it contain latex? \\
\hline \multirow{14}{*}{ Cefepime } & Cefepime 1 g F/A & $\mathrm{ABL}$ & No \\
\hline & Cefepime $2 \mathrm{~g}$ F/A & $\mathrm{ABL}$ & No \\
\hline & Cefepime $1 \mathrm{~g}$ F/A & Aurobindo & No \\
\hline & Cefepime 2 g F/A & Aurobindo & No \\
\hline & Cemax $1 \mathrm{~g}$ F/A & Instituto Biochimico & No \\
\hline & Cemax 2 g F/A & Instituto Biochimico & No \\
\hline & Cefepime 1 g F/A & Instituto Biochimico & No \\
\hline & Cefepime 2 g F/A & Instituto Biochimico & No \\
\hline & Cefepime 1 g F/A & Novafarma & No \\
\hline & Cefepime 2 g F/A & Novafarma & No \\
\hline & Cefepime 2 g F/A & Teuto & No \\
\hline & Clocef $1 \mathrm{~g}$ F/A & Teuto & No \\
\hline & Clocef 2 g F/A & Teuto & No \\
\hline & Unifepim 2 g F/A & União Química & No \\
\hline \multirow{5}{*}{ Ceftazidime } & Kefadim $1 \mathrm{~g}$ F/A & $\mathrm{ABL}$ & No \\
\hline & Ceftazidime $1 \mathrm{~g}$ F/A & Instituto Biochimico & No \\
\hline & Cefazima 1 g F/A & Instituto Biochimico & No \\
\hline & Ceftazidon $1 \mathrm{~g}$ F/A & Blau & No \\
\hline & Ceftafor $1 \mathrm{~g}$ F/A & Novafarma & No \\
\hline \multirow{7}{*}{ Ceftriaxone } & Ceftriaxone $1 \mathrm{~g}$ F/A & $\mathrm{ABL}$ & No \\
\hline & Keftron $1 \mathrm{~g}$ F/A & $\mathrm{ABL}$ & No \\
\hline & Ceftriaxone $1 \mathrm{~g}$ F/A & Blau & No \\
\hline & Triaxton 1 g F/A & Blau & No \\
\hline & Ceftriona $1 \mathrm{~g}$ F/A & Novafarma & No \\
\hline & Ceftriaxone $1 \mathrm{~g}$ F/A & Novafarma & No \\
\hline & Ceftriaxone $1 \mathrm{~g} F / \mathrm{A}$ & Teuto & No \\
\hline \multirow{4}{*}{ Cefuroxime } & Keroxime 750 mg F/A & $\mathrm{ABL}$ & No \\
\hline & Cefuroxime 750 mg F/A & Aurobindo & No \\
\hline & Cefuroxime 750 mg F/A & Instituto Biochimico & No \\
\hline & Monocef 750 mg F/A & Instituto Biochimico & No \\
\hline Cetuximab & Erbitux 5 mg/mL F/A 20 mL & Merck & No \\
\hline \multirow{9}{*}{ Ciprofloxacin } & Ciprofloxacin 2\% bag $100 \mathrm{~mL}$ & Fresenius Kabi & No \\
\hline & Ciprofloxacin 2\% bag 200 mL & Fresenius Kabi & No \\
\hline & Fresoflox 2\% bag 100 mL & Fresenius Kabi & No \\
\hline & Fresoflox $2 \%$ bag $200 \mathrm{~mL}$ & Fresenius Kabi & No \\
\hline & Ciprofloxacin 2 mg/mL bag 100 mL & Isofarma/ Halex Istar & No \\
\hline & Ciprofloxacin 2 mg/mL bag $200 \mathrm{~mL}$ & Isofarma/ Halex Istar & No \\
\hline & Hifloxan 2 mg/mL bag 100 mL & Isofarma/ Halex Istar & No \\
\hline & Hifloxan 2 mg/mL bag 200 mL & Isofarma/ Halex Istar & No \\
\hline & Hypoflox 2\% bag 100 mL & Hypofarma & No \\
\hline \multirow{6}{*}{ Cisplatin } & Citoplax 50 mg F/A & Bérgamo & No \\
\hline & Citoplax 10 mg F/A & Bérgamo & No \\
\hline & C-platin $10 \mathrm{mg} \mathrm{F} / \mathrm{A}$ & Blau & No \\
\hline & C-platin 50 mg F/A & Blau & No \\
\hline & Fauldcispla 50 mg F/A & Libbs & No \\
\hline & Fauldcispla 10 mg F/A & Libbs & No \\
\hline
\end{tabular}


Figure 2: Information about the presence of latex in standard injectable drugs.

\begin{tabular}{|c|c|c|c|}
\hline Drugs & Presentation & Manufacturer & Does it contain latex? \\
\hline \multirow{9}{*}{ Citarabine } & Citarabine 100 mg F/A & Accord & No \\
\hline & Citarabine 500 mg F/A & Accord & No \\
\hline & Cytos 100 mg F/A & Accord & No \\
\hline & Cytos 500 mg F/A & Accord & No \\
\hline & Citarax 100 mg F/A & Blau & No \\
\hline & Citarax 500 mg F/A & Blau & No \\
\hline & Fauldcita $500 \mathrm{mg}$ F/A & Libbs & No \\
\hline & Leustatin 8 mg F/A & Janssen-Cilag & No \\
\hline & Leustatin $10 \mathrm{mg} \mathrm{F} / \mathrm{A}$ & Janssen-Cilag & No \\
\hline \multirow{4}{*}{ Clarithromycin } & Klaricid 500 mg F/A & Abbott & No \\
\hline & Clarithromycin 500 mg F/A & Abbott & No \\
\hline & Clarithromycin 500 mg F/A & $A B L$ & No \\
\hline & Clarithromycin 500 mg F/A & MR Pharma & No \\
\hline Cloranfenicol & Arifenicol $1 \mathrm{~g} F / \mathrm{A}$ & Blau & No \\
\hline \multirow{2}{*}{ Cristalline penicillin } & Aricilina $5.000 .000 \mathrm{UI} \mathrm{F/A}$ & Blau & No \\
\hline & Cristacilina 5.000 .000 UI F/A & Novafarma & No \\
\hline \multirow{8}{*}{ Dacarbazine } & Dacarbazine 100 mg F/A & Bérgamo & No \\
\hline & Dacarbazine 200 mg F/A & Bérgamo & No \\
\hline & Dacarb 200 mg F/A & Eurofarma & No \\
\hline & Evodazin $100 \mathrm{mg}$ & Farmarin & No \\
\hline & Evodazin $200 \mathrm{mg}$ & Farmarin & No \\
\hline & Asercit $200 \mathrm{mg} F / \mathrm{A}$ & Fresenius Kabi & No \\
\hline & Asercit $100 \mathrm{mg} F / \mathrm{A}$ & Fresenius Kabi & No \\
\hline & Fauldacar 200 mg F/A & Libbs & No \\
\hline Daptomycin & Cubicin 500 mg F/A & MSD & No \\
\hline Daunorrubicin & Evoclass 20 mg F/A & Farmarin & No \\
\hline \multirow{4}{*}{ Dexmedetomidine } & Dexmedetomidine $100 \mathrm{mcg} / \mathrm{mL} / \mathrm{A} 2 \mathrm{~mL}$ & Aurobindo & No \\
\hline & Dexmedetomidine $100 \mathrm{mcg} / \mathrm{mL} \mathrm{F} / \mathrm{A} 2 \mathrm{~mL}$ & Eurofarma & No \\
\hline & Dexmedetomidine $100 \mathrm{mcg} / \mathrm{mL} \mathrm{F} / \mathrm{A} 2 \mathrm{~mL}$ & União Química & No \\
\hline & Extodin $100 \mathrm{mcg} / \mathrm{mL} \mathrm{F} / \mathrm{A} 2 \mathrm{~mL}$ & União Química & No \\
\hline Dextroketamine & Ketamin $50 \mathrm{mg} / \mathrm{mL}$ F/A $10 \mathrm{~mL}$ & Cristália & No \\
\hline \multirow{8}{*}{ Docetaxel } & Docetaxel 20 mg F/A & Sanofi & No \\
\hline & Docetaxel 80 mg F/A & Sanofi & No \\
\hline & Taxotere 20 mg F/A & Sanofi & No \\
\hline & Taxotere 80 mg F/A & Sanofi & No \\
\hline & Docetaxel 80 mg F/A & Glenmark & No \\
\hline & Docetaxel 20 mg F/A & Glenmark & No \\
\hline & Doceglennu 80 mg F/A & Glenmark & No \\
\hline & Doceglennu 20 mg F/A & Glenmark & No \\
\hline Ertapenem & Invanz $1 \mathrm{~g}$ F/A & MSD & No \\
\hline Esmolol & Brevibloc $10 \mathrm{mg} / \mathrm{mL} \mathrm{F} / \mathrm{A}$ & Cristália & No \\
\hline Etoposide & Eposide 20 mg/mL F/A 5 mL & Blau & No \\
\hline Fibrinogen & Fibrinogen $1 \mathrm{~g} F / \mathrm{A}$ & CSL Behring & No \\
\hline \multirow{2}{*}{ Filgrastim } & Filgrastim 300 mcg/mL F/A & Blau & No \\
\hline & Filgrastim $300 \mathrm{mcg} / \mathrm{mL} \mathrm{F} / \mathrm{A} 1 \mathrm{~mL}$ & Blau & No \\
\hline \multirow{4}{*}{ Fluconazole } & Fresolcan $2 \mathrm{mg} / \mathrm{mL}$ bag $100 \mathrm{~mL}$ & Fresenius Kabi & No \\
\hline & Fluconazole $2 \mathrm{mg} / \mathrm{mL}$ bag $250 \mathrm{~mL}$ & Fresenius Kabi & No \\
\hline & Fluconazole $2 \mathrm{mg} / \mathrm{mL}$ bag $100 \mathrm{~mL}$ & Isofarma/ Halex Istar & No \\
\hline & Hypoflucnazol 2 mg/mL F/A $100 \mathrm{~mL}$ & Hypofarma & No \\
\hline \multirow{2}{*}{ Fludarabine } & Evoflubine $50 \mathrm{mg} \mathrm{F} / \mathrm{A}$ & Farmarin & No \\
\hline & Fludalibbs $50 \mathrm{mg} \mathrm{F} / \mathrm{A}$ & Libbs & No \\
\hline Fluorescein & Fluorescein 20\% F/A $5 \mathrm{~mL}$ & Citopharma & No \\
\hline
\end{tabular}


Figure 2: Information about the presence of latex in standard injectable drugs.

\begin{tabular}{|c|c|c|c|}
\hline Drugs & Presentation & Manufacturer & Does it contain latex? \\
\hline \multirow{3}{*}{ Fluoruracil } & Flusan 50 mg/mL F/A 10 mL & Eurofarma & No \\
\hline & Fauldfluor 50 mg/mL F/A 10 mL & Libbs & No \\
\hline & Fauldfluor $50 \mathrm{mg} / \mathrm{mL}$ F/A $50 \mathrm{~mL}$ & Libbs & No \\
\hline \multirow{2}{*}{ Ganciclovir } & Cymevir $1 \mathrm{mg} / \mathrm{mL}$ bag $250 \mathrm{~mL}$ & Halex Istar & No \\
\hline & Cymevir $1 \mathrm{mg} / \mathrm{mL}$ bag $500 \mathrm{~mL}$ & Halex Istar & No \\
\hline \multirow{15}{*}{ Gencitabine } & Gencitabine $1 \mathrm{~g}$ F/A & Blau & No \\
\hline & Gencitabine 200 mg F/A & Blau & No \\
\hline & Gemcired $1 \mathrm{~g}$ F/A & Dr Reddy's & No \\
\hline & Gemcired 200 mg F/A & Dr Reddy's & No \\
\hline & Cytogem 200 mg F/A & Dr Reddy's & No \\
\hline & Gemzar 200 mg F/A & Eli Lilly & No \\
\hline & Gemzar 1 g F/A & Eli Lilly & No \\
\hline & Gencitabine $1 \mathrm{~g} F / \mathrm{A}$ & Eurofarma & No \\
\hline & Gencitabine 200 mg F/A & Eurofarma & No \\
\hline & Evozar $1 \mathrm{~g}$ F/A & Farmarin & No \\
\hline & Evozar 200 mg F/A & Farmarin & No \\
\hline & Genlibbs $1 \mathrm{~g}$ F/A & Libbs & No \\
\hline & Genlibbs 200 mg F/A & Libbs & No \\
\hline & Gemcit $1 \mathrm{~g}$ F/A & Sandoz & Yes \\
\hline & Gemcit 200 mg F/A & Sandoz & Yes \\
\hline \multirow{30}{*}{ Glucose } & Baxter glucose $100 \mathrm{mg} / \mathrm{mL}$ bag $250 \mathrm{~mL}$ & Baxter & No \\
\hline & Baxter glucose 100 mg/mL bag 500 mL & Baxter & No \\
\hline & Baxter glucose $50 \mathrm{mg} / \mathrm{mL}$ bag $250 \mathrm{~mL}$ & Baxter & No \\
\hline & Baxter glucose 50 mg/mL bag $500 \mathrm{~mL}$ & Baxter & No \\
\hline & Baxter glucose 50 mg/mL bag $100 \mathrm{~mL}$ & Baxter & No \\
\hline & Glucose Beker 50 mg/mL bag 100 mL & Beker & No \\
\hline & Glucose Beker 50 mg/mL bag $250 \mathrm{~mL}$ & Beker & No \\
\hline & Glucose Beker 50 mg/mL bag 500 mL & Beker & No \\
\hline & Glucose Beker 100 mg/mL bag 500 mL & Beker & No \\
\hline & Glucose Beker 100 mg/mL bag 250 mL & Beker & No \\
\hline & Glucose $50 \mathrm{mg} / \mathrm{mL}$ bag $100 \mathrm{~mL}$ & Equiplex & No \\
\hline & Glucose $50 \mathrm{mg} / \mathrm{mL}$ bag $250 \mathrm{~mL}$ & Equiplex & No \\
\hline & Glucose 50 mg/mL bag $500 \mathrm{~mL}$ & Equiplex & No \\
\hline & Glucose $5 \%$ bag $100 \mathrm{~mL}$ & Farmace & No \\
\hline & Glucose $5 \%$ bag $250 \mathrm{~mL}$ & Farmace & No \\
\hline & Glucose $5 \%$ bag $500 \mathrm{~mL}$ & Farmace & No \\
\hline & Glucose $5 \%$ bag $100 \mathrm{~mL}$ & Farmarin & No \\
\hline & Glucose $5 \%$ bag $500 \mathrm{~mL}$ & Farmarin & No \\
\hline & Glucose 100 mg/mL bag 500 mL & Fresenius Kabi & No \\
\hline & Glucose 100 mg/mL bag $250 \mathrm{~mL}$ & Fresenius Kabi & No \\
\hline & Glucose $50 \mathrm{mg} / \mathrm{mL}$ bag $100 \mathrm{~mL}$ & Fresenius Kabi & No \\
\hline & Glucose $50 \mathrm{mg} / \mathrm{mL}$ bag $250 \mathrm{~mL}$ & Fresenius Kabi & No \\
\hline & Glucose 50 mg/mL bag $500 \mathrm{~mL}$ & Fresenius Kabi & No \\
\hline & Glucose $5 \%$ bag $100 \mathrm{~mL}$ & Halex Istar & No \\
\hline & Glucose $10 \%$ bag $100 \mathrm{~mL}$ & Halex Istar & No \\
\hline & JP glucose 50 mg/mL bag $100 \mathrm{~mL}$ & JP Farma & No \\
\hline & JP glucose $50 \mathrm{mg} / \mathrm{mL}$ bag $250 \mathrm{~mL}$ & JP Farma & No \\
\hline & JP glucose 50 mg/mL bag 500 mL & JP Farma & No \\
\hline & JP glucose $100 \mathrm{mg} / \mathrm{mL}$ bag $250 \mathrm{~mL}$ & JP Farma & No \\
\hline & JP glucose 100 mg/mL bag 500 mL & JP Farma & No \\
\hline \multirow{2}{*}{ Hepatitis B vaccine } & Euvax 200 mcg HBcHG F/A 10 mL & Sanofi & No \\
\hline & Hepatitis B vaccine & Serum Institute of India & No \\
\hline \multirow{2}{*}{ Hidrocortisone } & Hidrocortisone 100 mg F/A & Blau & No \\
\hline & Hidrocortisone 500 mg F/A & Blau & No \\
\hline
\end{tabular}


Figure 2: Information about the presence of latex in standard injectable drugs.

\begin{tabular}{|c|c|c|c|}
\hline Drugs & Presentation & Manufacturer & Does it contain latex? \\
\hline \multirow{8}{*}{ Hidrocortisone } & Ariscorten 100 mg F/A & Blau & No \\
\hline & Ariscorten $500 \mathrm{mg} F / \mathrm{A}$ & Blau & No \\
\hline & Hidrocortisone $100 \mathrm{mg} F / \mathrm{A}$ & Blau & No \\
\hline & Hidrocortisone 500 mg F/A & Blau & No \\
\hline & Gliocort 100 mg F/A & Novafarma & No \\
\hline & Gliocort 500 mg F/A & Novafarma & No \\
\hline & Androcortil $100 \mathrm{mg}$ & Teuto & No \\
\hline & Androcortil $500 \mathrm{mg}$ & Teuto & No \\
\hline \multirow{7}{*}{ Human albumin } & Albiomin 20\% F/A $50 \mathrm{~mL}$ & Biotest & No \\
\hline & Albumax $20 \%$ F/A $50 \mathrm{~mL}$ & Blau & No \\
\hline & Balbimax 20\% F/A $50 \mathrm{~mL}$ & Blau & No \\
\hline & Alburex 20\% F/A 50 mL & CSL Behring & No \\
\hline & Beribumin $20 \% \mathrm{~F} / \mathrm{A} 50 \mathrm{~mL}$ & CSL Behring & No \\
\hline & Human albumin $20 \% \mathrm{~F} / \mathrm{A} 50 \mathrm{~mL}$ & Grifols & No \\
\hline & Octalbin $20 \% \mathrm{~F} / \mathrm{A} 50 \mathrm{~mL}$ & Octapharma & No \\
\hline \multirow{13}{*}{ Human immunoglobulin } & Intratec 5 g F/A 100 mL & Biotest & No \\
\hline & Intratec $2,5 \mathrm{~g}$ F/A $50 \mathrm{~mL}$ & Biotest & No \\
\hline & Pentaglobin $5 \mathrm{~g}$ F/A $100 \mathrm{~mL}$ & Biotest & No \\
\hline & Pentaglobin $2,5 \mathrm{~g} \mathrm{~F} / \mathrm{A} 50 \mathrm{~mL}$ & Biotest & No \\
\hline & Human immunoglobulin $5 \mathrm{~g}$ F/A & Blau & No \\
\hline & Immunoglobulin $5 \mathrm{~g}$ F/A $100 \mathrm{~mL}$ & Blau & No \\
\hline & Immunoglobulin 2,5 g F/A $50 \mathrm{~mL}$ & Blau & No \\
\hline & Flebogamma 5 g F/A 100 mL & Grifols & No \\
\hline & Flebogamma 2,5 g F/A $50 \mathrm{~mL}$ & Grifols & No \\
\hline & Igvena $5 \mathrm{~g} \mathrm{~F} / \mathrm{A} 100 \mathrm{~mL}$ & Kedrion & No \\
\hline & Igvena $2,5 \mathrm{~g}$ F/A $50 \mathrm{~mL}$ & Kedrion & No \\
\hline & Octagam $5 \mathrm{~g}$ F/A $100 \mathrm{~mL}$ & Octapharma & No \\
\hline & Octagam $2,5 \mathrm{~g} F / \mathrm{A} 50 \mathrm{~mL}$ & Octapharma & No \\
\hline \multirow{3}{*}{ Human NPH insulin } & Insunorm N $100 \mathrm{UI} / \mathrm{mL}$ F/A $10 \mathrm{~mL}$ & Aspen Pharma & No \\
\hline & Humulin N $100 \mathrm{UI} / \mathrm{mL}$ F/A $10 \mathrm{~mL}$ & Eli Lilly & Yes \\
\hline & Novolin N $100 \mathrm{UI} / \mathrm{mL}$ F/A $10 \mathrm{~mL}$ & Novo Nordisk & No \\
\hline \multirow{2}{*}{ Hydroxyethylamide } & Voluven $6 \%$ bag $500 \mathrm{~mL}$ & Fresenius Kabi & No \\
\hline & Plasmin $6 \%$ bag $500 \mathrm{~mL}$ & Halex Istar & No \\
\hline Idarubicin & Evomid $10 \mathrm{mg} F / \mathrm{A}$ & Farmarin & No \\
\hline \multirow{4}{*}{ Ifosfamide } & Ifosfamide $1 \mathrm{~g}$ F/A & Eurofarma & No \\
\hline & Ifosfamide $2 \mathrm{~g}$ F/A & Eurofarma & No \\
\hline & Ifosmide $1 \mathrm{~g} \mathrm{F/A}$ & Eurofarma & No \\
\hline & Evolox $1 \mathrm{~g} F / \mathrm{A}$ & Farmarin & No \\
\hline \multirow{3}{*}{ Imipenem + cilastatin } & Imipenem + cilastatin $500 \mathrm{mg}+500 \mathrm{mg} \mathrm{F} / \mathrm{A}$ & $A B L$ & No \\
\hline & Tiepém 500 mg + 500 mg F/A & Instituto Biochimico & No \\
\hline & Imipenem + cilastatin $500 \mathrm{mg}+500 \mathrm{mg} \mathrm{F} / \mathrm{A}$ & Instituto Biochimico & No \\
\hline \multirow{3}{*}{ Imipenem + cilastatin } & Imipenem + cilastatin 500 mg + $500 \mathrm{mg} F / \mathrm{A}$ & Midfarma & No \\
\hline & Tienan 500 mg + 500 mg F/A & MSD & Yes \\
\hline & Imicil 500 mg + 500 mg F/A & União Química & No \\
\hline \multirow{2}{*}{ Infliximab } & Infliximab $10 \mathrm{mg} / \mathrm{mL} \mathrm{F} / \mathrm{A}$ & Bio-Manguinhos & No \\
\hline & Remicade $10 \mathrm{mg} / \mathrm{mL}$ F/A $10 \mathrm{~mL}$ & Janssen-Cilag & No \\
\hline \multirow{6}{*}{ Insulin glargine } & Glargilin $100 \mathrm{UI} / \mathrm{mL}$ F/A $10 \mathrm{~mL}$ & Biomm & No \\
\hline & Glargilin $100 \mathrm{UI} / \mathrm{mL}$ F/A $3 \mathrm{~mL}$ & Biomm & No \\
\hline & Basaglar $100 \mathrm{UI} / \mathrm{mL} \mathrm{F} / \mathrm{A} 3 \mathrm{~mL}$ & Eli Lilly & Yes \\
\hline & Basaglar $100 \mathrm{UI} / \mathrm{mL}$ F/A $10 \mathrm{~mL}$ & Eli Lilly & Yes \\
\hline & Lantus $100 \mathrm{UI} / \mathrm{mL}$ F/A $10 \mathrm{~mL}$ & Sanofi & $\begin{array}{l}\text { No, but unable to ensure that during } \\
\text { manipulation no latex traces are found }\end{array}$ \\
\hline & Lantus $100 \mathrm{UI} / \mathrm{mL}$ F/A $3 \mathrm{~mL}$ & Sanofi & $\begin{array}{l}\text { No, but unable to ensure that during } \\
\text { manipulation no latex traces are found }\end{array}$ \\
\hline
\end{tabular}


Figure 2: Information about the presence of latex in standard injectable drugs.

\begin{tabular}{|c|c|c|c|}
\hline Drugs & Presentation & Manufacturer & Does it contain latex? \\
\hline \multirow{2}{*}{ Insulin lispro } & Humalog $100 \mathrm{UI} / \mathrm{mL}$ F/A $10 \mathrm{~mL}$ & Eli Lilly & Yes \\
\hline & Humalog $100 \mathrm{UI} / \mathrm{mL}$ F/A $3 \mathrm{~mL}$ & Eli Lilly & Yes \\
\hline \multirow{3}{*}{ Irinotecan } & Proto-itecan $20 \mathrm{mg} / \mathrm{mL}$ F/A $2 \mathrm{~mL}$ & $A B L$ & No \\
\hline & Irinotecano $20 \mathrm{mg} / \mathrm{mL}$ F/A $5 \mathrm{~mL}$ & $A B L$ & No \\
\hline & Irinotecano $20 \mathrm{mg} / \mathrm{mL} \mathrm{F} / \mathrm{A} 5 \mathrm{~mL}$ & Accord & No \\
\hline \multirow{4}{*}{ Irinotecan } & Tecnotecan 20 mg/mL F/A 2 mL & Zodiac & No \\
\hline & Tecnotecan $20 \mathrm{mg} / \mathrm{mL}$ F/A $5 \mathrm{~mL}$ & Zodiac & No \\
\hline & Evoterin $20 \mathrm{mg} / \mathrm{mL}$ F/A $5 \mathrm{~mL}$ & Farmarin & No \\
\hline & Irinotecano $20 \mathrm{mg} / \mathrm{mL} / \mathrm{F} 5 \mathrm{~mL}$ & Glenmark & No \\
\hline \multirow{4}{*}{ Ketoprofen } & Ketoprofen 100 mg F/A & Cristália & No \\
\hline & Ketoprofen 100 mg F/A & Eurofarma & No \\
\hline & Profenid 100 mg F/A & Sanofi & No \\
\hline & Artrinid $100 \mathrm{mg} F / \mathrm{A}$ & União Química & No \\
\hline Levosimendan & Simdax 2,5 mg F/A $5 \mathrm{~mL}$ & Biolab & No \\
\hline \multirow{2}{*}{ Lidocaine } & Lidocaine 2\% F/A $20 \mathrm{~mL}$ & Hipolabor & No \\
\hline & Lidocaine 2\% F/A 20 mL & Hypofarma & No \\
\hline Lidocaine with vasoconstrictor & Xylestesin 2\% F/A 20 mL & Cristália & No \\
\hline Lidocaine without vasoconstrictor & Xylestesin 2\% F/A $20 \mathrm{~mL}$ & Cristália & No \\
\hline Linezolide & Linezolide $2 \mathrm{mg} / \mathrm{mL}$ bag $300 \mathrm{~mL}$ & Beker & No \\
\hline Medroxiprogesterone & Demedrox 150 mg/mL F/A $1 \mathrm{~mL}$ & União Química & No \\
\hline \multirow{18}{*}{ Meropenem } & Meropenem 500 mg F/A & $A B L$ & No \\
\hline & Meropenem $1 \mathrm{~g}$ F/A & $A B L$ & No \\
\hline & Meropenem 500 mg F/A & Aurobindo & No \\
\hline & Meropenem $1 \mathrm{~g}$ F/A & Aurobindo & No \\
\hline & Mepenox 500 mg F/A & Instituto Biochimico & No \\
\hline & Mepenox 1 g F/A & Instituto Biochimico & No \\
\hline & Meropenem 500 mg F/A & Instituto Biochimico & No \\
\hline & Meropenem 1 g F/A & Instituto Biochimico & No \\
\hline & Meropenem 2 g F/A & Instituto Biochimico & No \\
\hline & Meromax $2 \mathrm{~g} F / \mathrm{A}$ & Eurofarma & No \\
\hline & Meropenem $1 \mathrm{~g}$ F/A & Eurofarma & No \\
\hline & Meropenem 500 mg F/A & Eurofarma & No \\
\hline & Meropenem $1 \mathrm{~g}$ F/A & Novafarma & No \\
\hline & Meropenem 500 mg F/A & Novafarma & No \\
\hline & Meropenem 500 mg F/A & Novafarma & No \\
\hline & Meropenem 1 g F/A & Novafarma & No \\
\hline & Meropenem 500 mg F/A & Teuto & No \\
\hline & Meropenem 1 g F/A & Teuto & No \\
\hline \multirow{13}{*}{ Methotrexate } & Hytas $25 \mathrm{mg} / \mathrm{mL}$ F/A $2 \mathrm{~mL}$ & Accord & No \\
\hline & Hytas $100 \mathrm{mg} / \mathrm{mL}$ F/A $5 \mathrm{~mL}$ & Accord & No \\
\hline & Hytas $100 \mathrm{mg} / \mathrm{mL}$ F/A $10 \mathrm{~mL}$ & Accord & No \\
\hline & Methotrexate $25 \mathrm{mg} / \mathrm{mL}$ F/A $20 \mathrm{~mL}$ & Blau & No \\
\hline & Methotrexate $100 \mathrm{mg} / \mathrm{mL}$ F/A $10 \mathrm{~mL}$ & Blau & No \\
\hline & Fauldmetro 25 mg/mL F/A 20 mL & Libbs & No \\
\hline & Fauldmetro $25 \mathrm{mg} / \mathrm{mL}$ F/A $2 \mathrm{~mL}$ & Libbs & No \\
\hline & Fauldmetro 25 mg/mL F/A 10 mL & Libbs & No \\
\hline & Fauldmetro 25 mg/mL F/A $50 \mathrm{~mL}$ & Libbs & No \\
\hline & Tevametho $25 \mathrm{mg} / \mathrm{mL}$ F/A $2 \mathrm{~mL}$ & Teva & No \\
\hline & Tevametho $25 \mathrm{mg} / \mathrm{mL}$ F/A $20 \mathrm{~mL}$ & Teva & No \\
\hline & Tevametho 25 mg/mL F/A 10 mL & Teva & No \\
\hline & Tevametho $25 \mathrm{mg} / \mathrm{mL} \mathrm{F} / \mathrm{A} 50 \mathrm{~mL}$ & Teva & No \\
\hline
\end{tabular}


Figure 2: Information about the presence of latex in standard injectable drugs.

\begin{tabular}{|c|c|c|c|}
\hline Drugs & Presentation & Manufacturer & Does it contain latex? \\
\hline \multirow{8}{*}{ Methylprednisolone } & Solupren $125 \mathrm{mg} F / \mathrm{A} 2 \mathrm{~mL}$ & Bérgamo & No \\
\hline & Solupren $50 \mathrm{mg} F / \mathrm{A} 8 \mathrm{~mL}$ & Bérgamo & No \\
\hline & Methylprednisolone 125 mg F/A 2 mL & Blau & No \\
\hline & Methylprednisolone 500 mg F/A 8 mL & Blau & No \\
\hline & Methylprednisolone 125 mg F/A 2 mL & Novafarma & No \\
\hline & Methylprednisolone 500 mg F/A 8 mL & Novafarma & No \\
\hline & Unimedrol 125 mg F/A 2 mL & União Química & No \\
\hline & Unimedrol 500 mg F/A 8 mL & União Química & No \\
\hline \multirow{11}{*}{ Metronidazole } & Metronidazole $5 \mathrm{mg} / \mathrm{mL}$ bag $100 \mathrm{~mL}$ & $A B L$ & No \\
\hline & Metronidazole $5 \mathrm{mg} / \mathrm{mL}$ bag $100 \mathrm{~mL}$ & Beker & No \\
\hline & Nidazofarma 5 mg/mL bag $100 \mathrm{~mL}$ & Farmace & No \\
\hline & Metronidazole $5 \mathrm{mg} / \mathrm{mL}$ bag $100 \mathrm{~mL}$ & Fresenius Kabi & No \\
\hline & Endonidazol 5 mg/mL bag 100 mL & Fresenius Kabi & No \\
\hline & Flagyl 5 mg/mL bag $100 \mathrm{~mL}$ & Sanofi & No \\
\hline & Metronidazole $5 \mathrm{mg} / \mathrm{mL}$ bag $100 \mathrm{~mL}$ & Isofarma/ Halex Istar & No \\
\hline & Hidazol 5 mg/mL bag $100 \mathrm{~mL}$ & Isofarma/ Halex Istar & No \\
\hline & Metronidazole $5 \mathrm{mg} / \mathrm{mL}$ bag $100 \mathrm{~mL}$ & Hypofarma & No \\
\hline & Metronidazole $5 \mathrm{mg} / \mathrm{mL}$ bag $100 \mathrm{~mL}$ & JP Farma & No \\
\hline & Metronidazole $5 \mathrm{mg} / \mathrm{mL}$ bag $100 \mathrm{~mL}$ & Equiplex & No \\
\hline \multirow{2}{*}{ Micafungin } & Mycamine 100 mg F/A & Astellas & $\begin{array}{l}\text { No, but unable to ensure that during } \\
\text { manipulation no latex traces are found }\end{array}$ \\
\hline & Mycamine 50 mg F/A & Astellas & $\begin{array}{l}\text { No, but unable to ensure that during } \\
\text { manipulation no latex traces are found }\end{array}$ \\
\hline Mitoxantrone & Evomixan $2 \mathrm{mg} / \mathrm{mL}$ F/A $10 \mathrm{~mL}$ & Farmarin & No \\
\hline \multirow{3}{*}{ Moxifloxacin } & Moxifloxacin 1,6 mg/mL bag $250 \mathrm{~mL}$ & Eurofarma & No \\
\hline & Praiva $1,6 \mathrm{mg} / \mathrm{mL}$ bag $250 \mathrm{~mL}$ & Eurofarma & No \\
\hline & Moxibacten $1,6 \mathrm{mg} / \mathrm{mL}$ bag $250 \mathrm{~mL}$ & Halex Istar & No \\
\hline $\begin{array}{l}\text { Nonionic low osmolality } \\
\text { iodinated contrast }\end{array}$ & Henetix 300 mg/mL F/A & Guerbet & No \\
\hline Omeprazole & Omeprazole 40 mg F/A & Blau & No \\
\hline \multirow{5}{*}{ Omeprazole } & Oprazon 40 mg F/A & Blau & No \\
\hline & Omeprazole 40 mg F/A & Cristália & No \\
\hline & Omeprazole 40 mg F/A & Eurofarma & No \\
\hline & Stomedini 40 mg F/A & União Química & No \\
\hline & Uniprazol 40 mg F/A & União Química & No \\
\hline \multirow{6}{*}{ Oxaciline } & Oxaciline 500 mg F/A & Aurobindo & No \\
\hline & Oxanon 500 mg F/A & Blau & No \\
\hline & Oxaciline 500 mg F/A & Blau & No \\
\hline & Oxacilil 500 mg F/A & Novafarma & No \\
\hline & Oxaciline 500 mg F/A & Novafarma & No \\
\hline & Oxaciline 500 mg F/A & Teuto & No \\
\hline \multirow{13}{*}{ Oxaliplatin } & Oxaliplatin 50 mg F/A & Bérgamo & No \\
\hline & Oxaliplatin 100 mg F/A & Bérgamo & No \\
\hline & Oxa-platin 50 mg F/A & Blau & No \\
\hline & Oxa-platin 100 mg F/A & Blau & No \\
\hline & Oxaliplatin 50 mg F/A & Blau & No \\
\hline & Oxaliplatin $100 \mathrm{mg}$ F/A & Blau & No \\
\hline & Oxaliplatin 50 mg F/A & Eurofarma & No \\
\hline & Oxaliplatin $100 \mathrm{mg} F / \mathrm{A}$ & Eurofarma & No \\
\hline & Evoxali 100 mg F/A & Farmarin & No \\
\hline & Evoxali 50 mg F/A & Farmarin & No \\
\hline & Eloxatin $5 \mathrm{mg} / \mathrm{mL}$ F/A $10 \mathrm{~mL}$ & Sanofi & No \\
\hline & Eloxatin $5 \mathrm{mg} / \mathrm{mL}$ F/A $20 \mathrm{~mL}$ & Sanofi & No \\
\hline & Eloxatin $5 \mathrm{mg} / \mathrm{mL}$ F/A $40 \mathrm{~mL}$ & Sanofi & No \\
\hline
\end{tabular}


Figure 2: Information about the presence of latex in standard injectable drugs.

\begin{tabular}{|c|c|c|c|}
\hline Drugs & Presentation & Manufacturer & Does it contain latex? \\
\hline \multirow{13}{*}{ Oxaliplatin } & Oxaliplatin 5 mg/mL F/A 10 mL & Sanofi & No \\
\hline & Oxaliplatin 5 mg/mL F/A 20 mL & Sanofi & No \\
\hline & Oxaliplatin $5 \mathrm{mg} / \mathrm{mL} \mathrm{F} / \mathrm{A} 40 \mathrm{~mL}$ & Sanofi & No \\
\hline & Oxaliplatin 50 mg F/A & Glenmark & No \\
\hline & Oxaliplatin 100 mg F/A & Glenmark & No \\
\hline & Oxalibbs 50 mg F/A & Libbs & No \\
\hline & Oxalibbs 100 mg F/A & Libbs & No \\
\hline & Oxalibbs 5 mg/mL F/A 10 mL & Libbs & No \\
\hline & Oxalibbs $5 \mathrm{mg} / \mathrm{mL}$ F/A $20 \mathrm{~mL}$ & Libbs & No \\
\hline & Tevaoxali $5 \mathrm{mg} / \mathrm{mL}$ F/A $10 \mathrm{~mL}$ & Teva & No \\
\hline & Tevaoxali $5 \mathrm{mg} / \mathrm{mL}$ F/A 20mL & Teva & No \\
\hline & Tevaoxali 5 mg/mL F/A 28mL & Teva & No \\
\hline & Tevaoxali $5 \mathrm{mg} / \mathrm{mL}$ F/A 40mL & Teva & No \\
\hline \multirow{40}{*}{ Paclitaxel } & Paclitaxel $6 \mathrm{mg} / \mathrm{mL}$ F/A $5 \mathrm{~mL}$ & Accord & No \\
\hline & Paclitaxel $6 \mathrm{mg} / \mathrm{mL} \mathrm{F} / \mathrm{A} 16,7 \mathrm{~mL}$ & Accord & No \\
\hline & Paclitaxel $6 \mathrm{mg} / \mathrm{mL}$ F/A $50 \mathrm{~mL}$ & Accord & No \\
\hline & Pantium $6 \mathrm{mg} / \mathrm{mL} / \mathrm{A} 5 \mathrm{~mL}$ & Accord & No \\
\hline & Pantium $6 \mathrm{mg} / \mathrm{mL}$ F/A 16,7 mL & Accord & No \\
\hline & Pantium $6 \mathrm{mg} / \mathrm{mL}$ F/A $50 \mathrm{~mL}$ & Accord & No \\
\hline & Akssus $6 \mathrm{mg} / \mathrm{mL} \mathrm{F} / \mathrm{A} 16,7 \mathrm{~mL}$ & Bérgamo & No \\
\hline & Taxilan 6 mg/mL F/A 16,7 mL & Bérgamo & No \\
\hline & Paclitaxel $6 \mathrm{mg} / \mathrm{mL}$ F/A $5 \mathrm{~mL}$ & Blau & No \\
\hline & Paclitaxel $6 \mathrm{mg} / \mathrm{mL}$ F/A 16,7 mL & Blau & No \\
\hline & Paclitaxel $6 \mathrm{mg} / \mathrm{mL} \mathrm{F} / \mathrm{A} 50 \mathrm{~mL}$ & Blau & No \\
\hline & Taxol 6mg/mL F/A $5 \mathrm{~mL}$ & Bristol-Myers Squibb & $\begin{array}{l}\text { No, but unable to ensure that during } \\
\text { manipulation no latex traces are found }\end{array}$ \\
\hline & Taxol 6mg/mL F/A 16,7 mL & Bristol-Myers Squibb & $\begin{array}{l}\text { No, but unable to ensure that during } \\
\text { manipulation no latex traces are found }\end{array}$ \\
\hline & Taxol 6mg/mL F/A 50 mL & Bristol-Myers Squibb & $\begin{array}{l}\text { No, but unable to ensure that during } \\
\text { manipulation no latex traces are found }\end{array}$ \\
\hline & Abraxane 100 mg F/A & Celgene & No \\
\hline & Paclired $6 \mathrm{mg} / \mathrm{mL}$ F/A 16,7 mL & Dr Reddy's & No \\
\hline & Paclired $6 \mathrm{mg} / \mathrm{mL}$ F/A 41,7 mL & Dr Reddy's & No \\
\hline & Paclired $6 \mathrm{mg} / \mathrm{mL}$ F/A $5 \mathrm{~mL}$ & Dr Reddy's & No \\
\hline & Parexel $6 \mathrm{mg} / \mathrm{mL}$ F/A $5 \mathrm{~mL}$ & Zodiac & No \\
\hline & Parexel $6 \mathrm{mg} / \mathrm{mL}$ F/A 16,7 mL & Zodiac & No \\
\hline & Parexel $6 \mathrm{mg} / \mathrm{mL}$ F/A $25 \mathrm{~mL}$ & Zodiac & No \\
\hline & Parexel $6 \mathrm{mg} / \mathrm{mL}$ F/A $50 \mathrm{~mL}$ & Zodiac & No \\
\hline & Evotaxel $6 \mathrm{mg} / \mathrm{mL} \mathrm{F} / \mathrm{A} 5 \mathrm{~mL}$ & Farmarin & No \\
\hline & Evotaxel $6 \mathrm{mg} / \mathrm{mL} \mathrm{F} / \mathrm{A} 16,7 \mathrm{~mL}$ & Farmarin & No \\
\hline & Evotaxel $6 \mathrm{mg} / \mathrm{mL}$ F/A $25 \mathrm{~mL}$ & Farmarin & No \\
\hline & Evotaxel $6 \mathrm{mg} / \mathrm{mL}$ F/A $50 \mathrm{~mL}$ & Farmarin & No \\
\hline & Paclitaxel $6 \mathrm{mg} / \mathrm{mL}$ F/A $5 \mathrm{~mL}$ & Fresenius Kabi & No \\
\hline & Paclitaxel $6 \mathrm{mg} / \mathrm{mL} \mathrm{F} / \mathrm{A} 16,7 \mathrm{~mL}$ & Fresenius Kabi & No \\
\hline & Paclitaxel $6 \mathrm{mg} / \mathrm{mL}$ F/A $50 \mathrm{~mL}$ & Fresenius Kabi & No \\
\hline & Paclitaxel $6 \mathrm{mg} / \mathrm{mL} 5 \mathrm{~mL}$ & Glenmark & No \\
\hline & Paclitaxel $6 \mathrm{mg} / \mathrm{mL} 16,7 \mathrm{~mL}$ & Glenmark & No \\
\hline & Paclitaxel $6 \mathrm{mg} / \mathrm{mL} 25 \mathrm{~mL}$ & Glenmark & No \\
\hline & Paclitaxel $6 \mathrm{mg} / \mathrm{mL} 50 \mathrm{~mL}$ & Glenmark & No \\
\hline & Ontax $6 \mathrm{mg} / \mathrm{mL} \mathrm{F} / \mathrm{A} 5 \mathrm{~mL}$ & Libbs & No \\
\hline & Ontax $6 \mathrm{mg} / \mathrm{mL}$ F/A 16,7 mL & Libbs & No \\
\hline & Ontax $6 \mathrm{mg} / \mathrm{mL}$ F/A $25 \mathrm{~mL}$ & Libbs & No \\
\hline & Tarvexol $6 \mathrm{mg} / \mathrm{mL}$ F/A $5 \mathrm{~mL}$ & Sandoz & No, but unable to ensure is latex-free \\
\hline & Tarvexol $6 \mathrm{mg} / \mathrm{mL}$ F/A 16,7 mL & Sandoz & No, but unable to ensure is latex-free \\
\hline & Paclitaxel 6 mg/mL F/A 5 mL & Sandoz & No, but unable to ensure is latex-free \\
\hline & Paclitaxel $6 \mathrm{mg} / \mathrm{mL} \mathrm{F} / \mathrm{A} 16,7 \mathrm{~mL}$ & Sandoz & No, but unable to ensure is latex-free \\
\hline
\end{tabular}


Figure 2: Information about the presence of latex in standard injectable drugs.

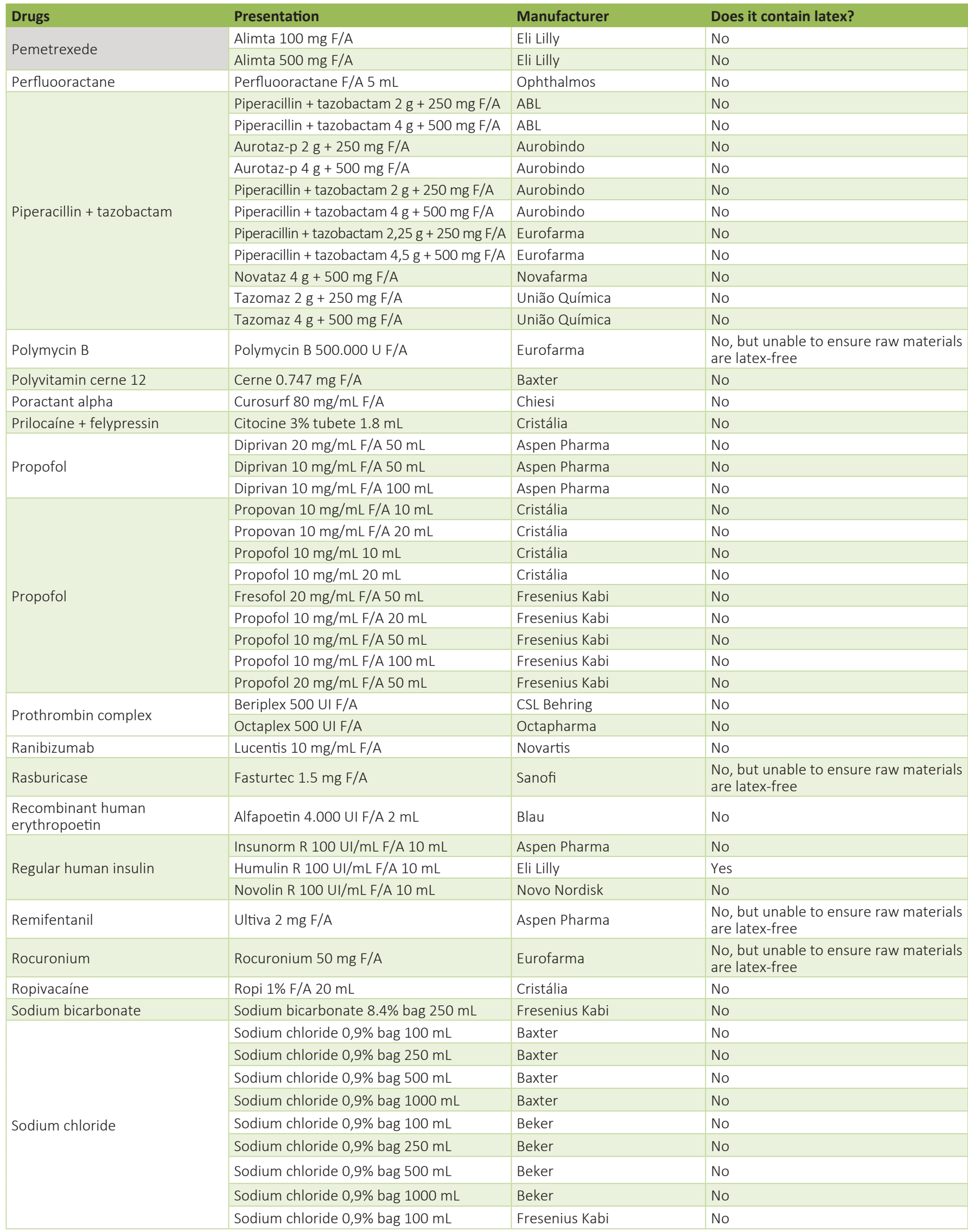


Figure 2: Information about the presence of latex in standard injectable drugs.

\begin{tabular}{|c|c|c|c|}
\hline Drugs & Presentation & Manufacturer & Does it contain latex? \\
\hline \multirow{4}{*}{ Sodium chloride } & Sodium chloride $0,9 \%$ bag $250 \mathrm{~mL}$ & Fresenius Kabi & No \\
\hline & Sodium chloride $0,9 \%$ bag $500 \mathrm{~mL}$ & Fresenius Kabi & No \\
\hline & Sodium chloride $0,9 \%$ bag $1000 \mathrm{~mL}$ & Fresenius Kabi & No \\
\hline & Sodium chloride $0,9 \%$ bag $100 \mathrm{~mL}$ & Halex Istar & No \\
\hline Sugammadex & Bridion 100 mg/mL F/A & MSD & No \\
\hline Suxamethonium & Succitrat 100 mg F/A & Blau & No \\
\hline \multirow[t]{2}{*}{ Swine heparin } & Hemofol $5.000 \mathrm{UI} / \mathrm{mL}$ F/A $5 \mathrm{~mL}$ & Cristália & No \\
\hline & Swine heparin 5.000 UI F/A 5 mL & União Química & No \\
\hline \multirow{4}{*}{ Teicoplanin } & Bactomax 200 mg F/A & Cristália & No \\
\hline & Bactomax $400 \mathrm{mg} F / \mathrm{A}$ & Cristália & No \\
\hline & Teicoplanin 200 mg F/A & Eurofarma & No \\
\hline & Teicoplanin 400 mg F/A & Eurofarma & No \\
\hline \multirow{4}{*}{ Teicoplanin } & Targocid 400 mg F/A & Sanofi & No \\
\hline & Teicoplanin 400 mg F/A & Teuto & No \\
\hline & Teicoplanin 400 mg F/A & União Química & No \\
\hline & Teicoplanin 200 mg F/A & União Química & No \\
\hline Terlipressin & Glypressin 1 mg F/A & Laboratórios Ferring & No \\
\hline Thiopental & Thiopentax $1 \mathrm{~g}$ F/A & Cristália & No \\
\hline Trastuzumab & Herceptin 150 mg F/A & Roche & No, but unable to ensure is latex-free \\
\hline Triancinolone & Triancinolone 40 mg/mL F/A $1 \mathrm{~mL}$ & Ophthalmos & No \\
\hline \multirow{2}{*}{ Trypan blue } & Trypan blue 0.1 \% F/A $1 \mathrm{~mL}$ & Oftalmopharma & No \\
\hline & Trypan blue 0.1 \% F/A 1 mL & Ophthalmos & No \\
\hline \multirow{5}{*}{ Vancomycin } & Vancomycin 500 mg F/A & $A B L$ & No \\
\hline & Vancomycin 500 mg F/A & Blau & No \\
\hline & Vancoson 500 mg F/A & Blau & No \\
\hline & Vancomycin 500 mg F/A & Teuto & No \\
\hline & Vancotrat $500 \mathrm{mg} F / \mathrm{A}$ & União Química & No \\
\hline Vecuronium & Vecuron 4 mg F/A & Cristália & No \\
\hline \multirow{2}{*}{ Vimblastine } & Rabinefil 10 mg F/A & Fresenius Kabi & No \\
\hline & Faulblastina 10 mg F/A & Libbs & No \\
\hline \multirow{3}{*}{ Vincristine } & Dabaz $1 \mathrm{mg} / \mathrm{mL}$ F/A $2 \mathrm{~mL}$ & Accord & No \\
\hline & Vincristine $1 \mathrm{mg} / \mathrm{mL} \mathrm{F} / \mathrm{A} 2 \mathrm{~mL}$ & Accord & No \\
\hline & Fauldvincri $1 \mathrm{mg} / \mathrm{mL} \mathrm{F} / \mathrm{A}$ & Libbs & No \\
\hline \multirow{2}{*}{ Voriconazole } & Micend 200 mg F/A & Bérgamo & No \\
\hline & Veac 200 mg F/A & Eurofarma & No \\
\hline \multirow{3}{*}{ Water for injection } & Water for injection bag $250 \mathrm{~mL}$ & Fresenius Kabi & No \\
\hline & Water for injection bag $500 \mathrm{~mL}$ & Fresenius Kabi & No \\
\hline & Water for injection bag $1000 \mathrm{~mL}$ & Fresenius Kabi & No \\
\hline \multirow{2}{*}{ Zoledronic acid } & Zoledronic acid $4 \mathrm{mg}$ F/A $5 \mathrm{~mL}$ & Sandoz & No \\
\hline & Zometa 4 mg/mL F/A 5 mL & Novartis & No \\
\hline
\end{tabular}

Figure 3. Classification of injectable drugs according to the presence or absence of latex $(n=123)$.

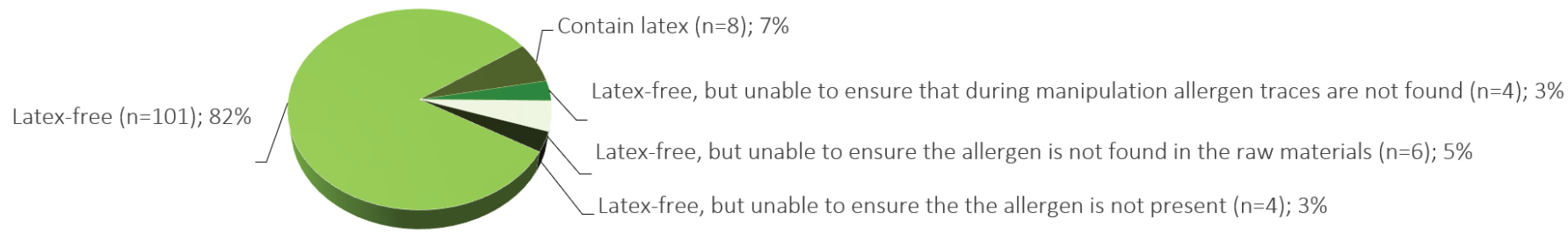


Figure 4. Injectable drugs with latex or at any risk of contamination.

\begin{tabular}{|c|c|c|}
\hline Drugs & Presentation & Manufacturer \\
\hline \multicolumn{3}{|l|}{ Contain latex } \\
\hline \multirow{2}{*}{ Alteplase } & Actilyse 10 mg F/A & \multirow{2}{*}{ Boehringer Ingelheim } \\
\hline & Actilyse 50 mg F/A & \\
\hline \multirow{2}{*}{ Gencitabine } & Gemcit $1 \mathrm{~g}$ F/A & \multirow{2}{*}{ Sandoz } \\
\hline & Gemcit 200 mg F/A & \\
\hline Imipenem + cilastatin & Tienan 500 mg + 500 mg F/A & MSD \\
\hline Anti-RhO (D) immunoglobulin & KamRhO-D 300 mcg F/A 2 mL & Panamerican Medical Supply \\
\hline \multirow{2}{*}{ Insulin glargin } & Basaglar $100 \mathrm{UI} / \mathrm{mL}$ F/A 3 mL & \multirow{2}{*}{ Eli Lilly } \\
\hline & Basaglar $100 \mathrm{UI} / \mathrm{mL}$ F/A $10 \mathrm{~mL}$ & \\
\hline Regular human insulin & Humulin R $100 \mathrm{UI} / \mathrm{mL}$ F/A $10 \mathrm{~mL}$ & Eli Lilly \\
\hline Human NPH insulin & Humulin N $100 \mathrm{UI} / \mathrm{mL}$ F/A $10 \mathrm{~mL}$ & Eli Lilly \\
\hline \multirow{2}{*}{ Insulin lispro } & Humalog $100 \mathrm{UI} / \mathrm{mL}$ F/A $10 \mathrm{~mL}$ & \multirow{2}{*}{ Eli Lilly } \\
\hline & Humalog 100 UI/mL F/A 3 mL & \\
\hline
\end{tabular}

\begin{tabular}{|c|c|c|}
\hline \multicolumn{3}{|c|}{ Latex-free, but unable to ensure that during the manipulation no traces of the allergen are found } \\
\hline \multirow{2}{*}{ Insulin glargin } & Lantus $100 \mathrm{UI} / \mathrm{mL}$ F/A $3 \mathrm{~mL}$ & \multirow{2}{*}{ Sanofi } \\
\hline & Lantus $100 \mathrm{UI} / \mathrm{mL}$ F/A $10 \mathrm{~mL}$ & \\
\hline \multirow{2}{*}{ Micafungin } & Mycamine 100 mg F/A & \multirow{2}{*}{ Astellas } \\
\hline & Mycamine 50 mg F/A & \\
\hline \multirow{3}{*}{ Paclitaxel } & Taxol $6 \mathrm{mg} / \mathrm{mL} F / \mathrm{A} 5 \mathrm{~mL}$ & \multirow{3}{*}{ Bristol-Myers Squibb } \\
\hline & Taxol 6 mg/mL F/A 16,7 mL & \\
\hline & Taxol 6 mg/mL F/A $50 \mathrm{~mL}$ & \\
\hline Botulinum toxin A & Botox $100 \mathrm{UI} F / \mathrm{A}$ & Allergan \\
\hline \multicolumn{3}{|c|}{ Latex-free, but unable to ensure that raw materials contain the allergen } \\
\hline Amoxicillin + clavulanate & Amoxicillin + clavulanate $1 \mathrm{~g}+200 \mathrm{mg} F / \mathrm{A}$ & Eurofarma \\
\hline Benzathine benzylpenicillin & Benzetacil 1.200.000 UI F/A & Eurofarma \\
\hline Polymycin B & Polymycin B 500.000 U F/A & Eurofarma \\
\hline Rasburicase & Fasturtec $1,5 \mathrm{mg} \mathrm{F} / \mathrm{A}$ & Sanofi \\
\hline Remifentanil & Ultiva 2 mg F/A & Aspen Pharma \\
\hline Rocuronium & Rocuronium $50 \mathrm{mg} F / \mathrm{A}$ & Eurofarma \\
\hline \multicolumn{3}{|c|}{ Latex-free, but unable to ensure whether allergen-free } \\
\hline Bevacizumab & Avastin $25 \mathrm{mg} / \mathrm{mL} \mathrm{F} / \mathrm{A} 4 \mathrm{~mL}$ & Roche \\
\hline Ceftriaxone & Rocefin $1 \mathrm{~g} F / \mathrm{A}$ & Roche \\
\hline \multirow{4}{*}{ Paclitaxel } & Tarvexol $6 \mathrm{mg} / \mathrm{mL} \mathrm{F} / \mathrm{A} 5 \mathrm{~mL}$ & \multirow{4}{*}{ Sandoz } \\
\hline & Tarvexol 6 mg/mL F/A 16,7 mL & \\
\hline & Paclitaxel $6 \mathrm{mg} / \mathrm{mL}$ F/A $5 \mathrm{~mL}$ & \\
\hline & Paclitaxel $6 \mathrm{mg} / \mathrm{mL}$ F/A 16,7 ML & \\
\hline Trastuzumab & Herceptin 150 mg F/A & Roche \\
\hline
\end{tabular}

\section{Discussion}

In 1999, the American Society of Anesthesiology suggested some measures of care and prevention as the correct identification of the allergic patient, elective surgeries carried out as early as possible to minimize latex antigens levels as aerosol in the operation room, surveys and listing of all the institution's products containing latex. ${ }^{10}$

There was an expressive reduction of the use of products and medical devices with latex in the last years, due to the increase of allergic reactions in health professionals and patients, considered the second cause of anaphylactic reactions in the surgeryanesthetic area. $6,23,24$ The intensity of these reactions that can range from dermatological findings to shock and anaphylaxis have been promoting efforts in hospital services worldwide in favor of the substitution of their materials and products by other latexfree..$^{17}$ According to Heitz \& Bader (2010), the American database latexdrugs.com conducted a survey which showed that $78 \%$ of the drugs were latex-free, $14 \%$ had traces of the allergen and $8 \%$ failed to be classified as latex-free drugs, because their packages or part of the production batches contained latex or would be latex-free in the future. ${ }^{25}$ These data corroborate our findings, since few products contain latex in their composition.

Some manufacturers had difficulties in accessing the information requested immediately as the composition of the rubber stopper utilized in the primary product package. Some responses were received only after one month or follow up, e-mails or telephone contact. The delay to respond these information is concerning as the demand for this is urgent when related to the immediate use in the hospital.

The manufacturers justified the delay because the product is 
manufactured abroad which demands contact with foreign employees to confirm the information, lack of quick access information in the customer "contact us" option and necessity to check with third parties like the manufacturers of raw materials used in the medications. Most of the data received was sent by the manufacturers informed in e-mail body. Internal documents from the companies were made available to the study team, including analyzes of the presence or absence of the allergen. However, these documents are not public, and are unavailable on the companies' websites, ANVISA leaflets, or in medicines package inserts. The study results were not registered in any database.

The literature corroborates the difficulty of access to manufacturer data about the composition of the rubber stopper. A study whose objective was to identify medications with latex in 2008 reported industry's response time over one month. We received the same justification in our study, the need to contact international officers to get responses. Berbare et al. found manufacturers who did not have the information about the composition of injectable medication vial stoppers. The sanitary authorities should evaluate the presence and identification of packages with latex. ${ }^{21}$

Whereas the severity of latex-induced allergic reactions, a significant worsening of the patient outcome could occur if the presence of the allergen in drugs is not clearly stated. In cases where only one industry sells the drug, if the patient is allergic to latex, its use would be unfeasible. ${ }^{14}$

The United States Food and Drug Administration (FDA) issued guidelines for the identification of medical products to inform the users that the product contains latex. ${ }^{26}$ In 1998, Holzman \& Katz warned about the importance of labeling products with latex as protective additional measure. ${ }^{27}$ All these measures are justified due to contamination with the allergen that may occur when the liquid medication comes into contact with the stopper before or after the needle is pushed through the latex seal. ${ }^{28}$ The pharmaceutical industries should include information about the presence of latex in package inserts and labels to help health professionals and patients at the end of the medicine distribution chain.

Although the list of drugs do not reflect the reality of all Brazilian hospitals, it was possible to highlight the lack of identification of medications that contain the allergen in their composition or primary packaging. It is necessary that health institutions develop a contingency plan for the identification of these drugs within hospital units, in order to avoid adverse events. Our difficulty during data collection about the presence of latex also shows that pharmaceutical industries need a strategic planning to answer questions from health professionals and patients, in addition to including latex information in patient package insert and on packaging.

\section{Conclusion}

Access to information is difficult and scarce data in the scientific literature, package inserts and packaging about the presence of latex in drugs are a concrete issue which affects health professionals and patients. It is essential that the interdisciplinary team is aware of this information rapidly, to enhance the patient's safety during different procedures and phases of care for better choice of therapeutic options and possible replacements safely. Sanitary authorities and other responsible bodies need to carefully watch the composition of drugs to evaluate whether information such as the presence of latex in package inserts and packaging should be mandatory. Data about the composition of drugs should not be restricted to the active principle and its excipients alone, they must encompass the entire medications production and commercialization chain.

Although the information gathered does not reflect the reality of all Brazilian hospitals, they may help other health services to promote their patients' safety because their content can be accessed immediately.

Due to the relevance of the data collected, they were included in the Pharmacotherapy Guideline of Hospital Federal dos Servidores do Estado to facilitate the access to information for interdisciplinary team. Identification labels informing the presence of latex in hospital products allows the prompt visualization and aims to help preventing possible errors that can lead to unfavorable clinical outcomes.

\section{Funding}

The present study has not received any sponsorship, funding, or resources from third party.

\section{Collaborators}

Conception and design: RGP, APA. Analysis and interpretation of data: RGP, MCS, BBO, LPG, IGH, APA. Writing of the article: RGP, MCS, BBO, LPG, IGH, APA. Relevant critical review of the intellectual content: APA, RTA. English Translator: JCC.

\section{Conflict of interest statement}

The authors declare that there are no conflicts of interest regarding this article.

\section{References}

1. Wu M, Mcintosh J, Liu J. Current prevalence rate of latex allergy: Why it remains a problem? J Occup Health. 2016;58(2):138-44. DOI: https://doi.org/10.1539/joh.150275-RA.

2. Sá $A B$, Mallozi MC, Solé D. Alergia ao látex: atualização. Rev Bras Alerg Imunopatol. 2010;33(5):173-83. DOI: https:// doi.org/0103-2259/10/33-05/173.

3. Gomes MJ, Barbosa RS, Dias FP, et al. Sensitivity to latex and the dosage of specific antibodies in professionals in the area of health. Ciênc Saúde Coletiva. 2010;17(2):351-8. DOI: http://dx.doi.org/10.1590/S1413-81232012000200009.

4. Santos RMSF. Assistência de enfermagem perioperatória a pacientes alérgicos ao látex [dissertação]. Campinas, Universidade Estadual de Campinas, 2008. Available in: http:// repositorio.unicamp.br/jspui/handle/REPOSIP/309525. Accessed on: 6th Jun 2021.

5. Binkley HM, Schroyer T, Catalfano J. Latex allergies: a review of recognition, evaluation, management, prevention, education, and alternative product use. J Athl Train.2003;38(2):133-40. 
6. American College of Allergy, Asthma and Immunology (ACAAI). Alergia ao Látex. Available in: https://acaai.org/allergies/types/latex-allergy. Accessed on: 6th Jun 2021.

7. Adamson $\mathrm{M}$, Nadra A. Latex in medicine vial stoppers. Anaesthesia.2014;69:281-90. DOI: https://doi.org/10.1111/ anae.12610

8. Correa NB. Alergia ao Látex em profissional da Saúde. Revista Médica HSE.1999;33:1-4. Available in: http://www.hse.rj. saude.gov.br/profissional/revista/33/latex.asp. Accessed on: 6th Jun 2021.

9. Centers for Disease Control and Prevention. Latex in vaccine packaging. 2019. Available in: https://www.cdc.gov/vaccines/ pubs/pinkbook/downloads/appendices/B/latex-table.pdf. Accessed on: 6th Jun 2021.

10. Allarcon JB, Malito $\mathrm{M}$, Linde $\mathrm{H}$, et al. Alergia ao Látex. Rev Bras Anestesiol. 2003;53(1):89-96. Available in: https://www. scielo.br/pdf/rba/v53n1/v53n1a12.pdf. Accessed on: 6th Jun 2021.

11. Montalvão LN, Pires MC, Mello JF. Alergia ao látex em profissionais de saúde de São Paulo, Brasil. 2008;83(3):213-20. DOI: https://doi.org/10.1590/S0365-05962008000300004.

12. Fernandes $\mathrm{C}$, Silva $\mathrm{CMO}$, Segundo GRS. Prevalência de alergia ao látex IgE-mediada em um hospital universitário. Braz J Allergy Immunol. 2014;2(6):235-40. Available in: http:// aaai-asbai.org.br/detalhe_artigo.asp?id=711. Accessed on: 28th Oct 2021.

13. Yeh WSC, Kiohara PR, Soares ISC, et al. Prevalence of sensitivity signals to latex in meningomyelocele patients undergoing multiple surgical procedures. Rev Bras Anestesiol. 2012;62(1):56-62. DOI: https://doi.org/10.1590/ S0034-70942012000100008.

14. Sá AB, Araujo RFC, Cavalheiro S, et al. Profile of Latex Sensitization and Allergies in Children and Adolescents With Myelomeningocele in São Paulo, Brazil. J Investig Allergol Clin Immunol.2013;23(1):43-9. Available in: http://www.jiaci.org/ issues/vol23issue1/7.pdf. Accessed on: 28th Oct 2021.

15. Garro LS, Aun MV, Motta AA, et al. IgE, IgG4 e IgA específicas na alergia ao látex. Arq Asma Alerg Imunol.2017;1(1):99-108. DOI: http://dx.doi.org/10.5935/2526-5393.20170012.

16. Brasil. Agência Nacional de Vigilância Sanitária. Implantação do Núcleo de Segurança do Paciente em Serviços de Saúde. Série - Segurança do Paciente e Qualidade em Serviços de Saúde, 2016. Available in: https://www.saude. go.gov.br/images/imagens_migradas/upload/arquivos/2017-09/2016-anvisa---caderno-6---implantacao-nucleo-de-seguranca.pdf. Accessed on: 21th Apr 2020.

17. Brasil. Ministério da Saúde. Anexo 3: Protocolo de segurança na prescrição, uso e administração de medicamentos. Available in: https://portaldeboaspraticas.iff.fiocruz.br/wp-content/uploads/2020/01/protoc_identificacaoPaciente.pdf. Accessed on: 6th Jun 2021

18. United States. Department of Health and Human Services. NIOSH Alert: Preventing Allergic Reactions to Natural Rubber Latex in the Workplace. DHHS (NIOSH).1998:97-135. Available in: https://www.cdc.gov/niosh/docs/97-135/pdfs/97135. . $d f ?$ id $=10.26616 /$ NIOSHPUB97135 Accessed on: 6th Jun 2021.
19. Brasil. Presidência da República. Casa Civil. Lei №12.849, de 2 de agosto de 2013. Available in: https://www.planalto.gov.br/ ccivil_03/_ato2011-2014/2013/lei/l12849.htm. Accessed on: 28th Oct 2021.

20. Brasil. Ministério da Saúde. Agência Nacional de Vigilância Sanitária. RDC №26, de 02 de julho de 2015. Available in: https://bvsms.saude.gov.br/bvs/saudelegis/anvisa/2015/ rdc0026_26_06_2015.pdf. Accessed on: 28th Oct 2021.

21. Berbare MHAO, Jesus PG, Ferracini FT, et al. Safety of medicine therapy in latex-allergic patients. Einstein.2008;6(1):13-9. Available in: https://www.researchgate.net/publication/26539984_Safety_of_medicine_therapy_in_latex-allergic_patients. Accessed on: 6th Jun 2021.

22. Brasil. Agência Nacional de Vigilância Sanitária. Consultas. Available in: https://consultas.anvisa.gov.br/\#/medicamentos/. Accessed on: 6th Jun 2021.

23. Liberman P. Anaphylactic reaction during surgical and medical procedures. J Allergy Clin Immunol.2002;110:S64-9. DOI: https://doi.org/10.1067/mai.2002.124970.

24. Draisci G, Nucera E, Pollastrini E, et al. Anaphylactic reactions during cesarean section. IJOA.2007;16(1):63-7. DOI: https:// doi.org/10.1016/j.ijoa.2006.08.006.

25. Heitz JW; Bader SO. An evidence-based approach to medication for the surgical patient at risk for latex allergy: is it time to stop being stopper poppers? I Clin Ane.2010;22:477-83. DOI: https://doi.org/10.1016/j.jclinane.2009.12.006.

26. United States. United States Food and Medicine Administration. Guidance for Industry. User labeling for devices that contain natural rubber (21 CFR 801.437); Small entity compliance guide; guidance for industry. Available in: https://www. fda.gov/media/71135/download. Accessed on: 6th Jun 2021.

27. Batti MACSB. Alergia ao látex. Rev Bras Anestesiol.2003;53(5):555-60. Available in: https://www.scielo.br/ pdf/rba/v53n5/v53n5a01.pdf. Accessed on: 6th Jun 2021.

28. Association of Sirurgical Technologists. Guidelines for best practices for the natural rubber latex allergic and metal allergic patient. Available in: https://www.ast.org/uploadedFiles/ Main_Site/Content/About_Us/Guideline_Latex_Allergy.pdf. Accessed on:6th Jun 2021. 